\title{
Transición social en niñas, niños, niñes y adolescentes con incongruencia de género: revisión y sugerencias de intervención a profesionales
}

\section{Social transition in children and adolescents with gender incongruence: review and intervention recommendations for professionals}

\author{
Dorca Retamal Parra ${ }^{1,2,3^{*}}$ Wladimir Hermosilla Rubio ${ }^{2,3}$ Miguel Medel Arriagada ${ }^{1,2,3}$ Catalina Retamal Vargas ${ }^{4}$
}

\begin{abstract}
Resumen:
Introducción: la transición social en niñas (os, es) y adolescentes con incongruencia de género, es una intervención no médica, completamente reversible, que genera beneficios en la salud mental, pero que ha sido poco estudiada. El objetivo es realizar una revisión bibliográfica al respecto y desde ahí derivar recomendaciones a las(os) profesionales que intervienen en el apoyo a estas (os, es) niñas (os, es), adolescentes y sus familias. Métodos: II presente trabajo corresponde a una revisión entre los años 2010 - 2021 en revistas científicas en habla inglesa e hispana indexadas en PUBMED, SciELO y en Google Académico. Resultados: se describe la trayectoria en el desarrollo de la incongruencia de género. En las (os, es) prepúberes el interés está centrado en la persistencia y desistencia de la incongruencia de género y las distintas trayectorias. La transición social y la persistencia se pueden manifestar diferencialmente para niñas y niños trans. Realizar transición social en niñas (os, es) que lo solicitan favorece la salud mental. La familia y la comunidad viven sus propias transiciones. En Chile se han ido dando las condiciones en salud, educación y a nivel legislativo para apoyar la afirmación de género de las niñas (os, es) y adolescentes, pero falta educación respecto a la transición social en infancia y adolescencia. Conclusiones: dado los beneficios que genera la transición social en niñas (os, es) y adolescentes transgénero, se requiere que las(os) profesionales se eduquen e intervengan adecuadamente.
\end{abstract}

Palabras clave: transición social; transgénero; niñas(os); adolescentes; familia.

\begin{abstract}
:
Introduction: Social transition for boys, girls, and adolescents with gender incongruence is a completely reversible non-medical intervention that generates important mental health benefits, but reviews lack studies. The objective here is to do a bibliographic review regarding the matter, and from there, formulate recommendations for professionals who intervene to support these boys, girls, and adolescents, as well as their families. Method: This work reviews English and Spanish journals, published between 2010 and 2020 , indexed in PUBMED, SciELO, and Google Scholar. Results: The trajectory of the development of gender incongruence is described. For children in the 're-puberty stage, the interest is focused on the persistence and desistence of gender incongruence. Social transition and persistence can manifest differentially for trans boys and trans girls. Making a social transition for the boys and girls who request it benefits their mental health. The family and the community undergo their transformations. In Chile, conditions at a health, education, and legislative level, to support the gender affirmation of boys, girls and adolescents, have appeared. Still, there is a lack of education regarding the social transition in childhood and adolescence. Conclusions: Given the benefits that social transition generates for transgender boys, girls, and adolescents, it is required that professionals educate themselves and intervene accordingly.
\end{abstract}

Keywords: social transition; transgender; children; adolescents; family.

Fecha de envío: 2021-03-12 - Fecha de aceptación: 2021-06-21

(1) Equipo transpediátrico, Hospital las Higueras, Talcahuano, Chile.

(2) Equipo psiquiatría infantil, Hospital Las Higueras, Talcahuano, Chile.

(3) Programa de residentes de psiquiatría infantil de la UCSC, Talcahuano, Chile

(4) Facultad de medicina, Universidad de Concepción, Concepción, Chile

*Autor de correspondencia:dorca2003@hotmail.com. 


\section{Introducción}

La incongruencia de género es una marcada y persistente discordancia entre la identidad de género y el sexo asignado al nacer, lo que puede o no causar disforia o estrés en algunas personas. La Organización Mundial de la Salud (OMS) en el año 2018 retiró el témino transexualidad de la lista de enfermedades de salud mental y lo reemplazó por incongruencia de género en la lista de condiciones relacionadas con la salud sexual (Organización mundial de la Salud, 2018).

En Chile no existen estudios de prevalencia y los que se han realizado en EEUU estiman que el $0,5 \%$ de la población se podría definir con incongruencia de género; de esta manera haciendo una extrapolación de estas cifras a la población chilena del último censo, se estimaría que habrían más de 80000 personas con incongruencia de género y cada año existirían cerca de 30 personas que podrían solicitar asistencia sanitaria (Zapata et al., 2019).

Para otorgar una asistencia de calidad, es necesario que las (os) profesionales estén educadas (os), orienten y deriven adecuadamente. En un estudio chileno (Zapata et al., 2021), se exploró el conocimiento por parte de medicas(os) respecto de la atención en salud de personas con incongruencia de género, el 56\% había atendido a una persona transgénero en sus prácticas clínicas. El 95\% afirmo apoyo o empatía por estas (os, es) usuarias (os, es), mientras que el 4,3\% afirmó rechazo. Si bien es cierto que un porcentaje mayoritario apoya a esta población, un 12\% de las (os) médicas(os) estuvo en contra de usar intervenciones hormonales en menores de edad (Zapata et al., 2021), lo que lleva a considerar necesario una mayor educación, ya que el desconocimiento y/o los prejuicios pueden interferir el proceso de transición de una (un) niña (o, e) y adolescente.

El enfoque afirmativo en personas con incongruencia de género, considera tres tipos de intervenciones. En primer lugar, están las reversibles que involucran procedimientos no médicos a través de la transición social y otros de carácter médico por medio del bloqueo puberal con análogos de la hormona liberadora de gonadotropina. En segundo lugar, las intervenciones parcialmente reversibles son las que incorporan las terapias hormonales cruzadas, ya sea con estrógenos o testosterona utilizadas desde los 16 años en adelante. (Edwards-Leeper et al., 2016; Kaltiala-Heino et al., 2018; Turban et al., 2018). Finalmente, están las que son de carácter irreversible y que son consideradas así por tratarse de cirugías de reasignación de sexo realizadas después de los
18 años, siempre teniendo en consideración la fertilidad futura (Edwards-Leeper et al., 2016; Kaltiala-Heino et al., 2018; Turban et al., 2018).

En este artículo se desarrollará la primera intervención en el proceso afirmativo, específicamente la transición social, considerada una intervención no médica y relacionada a cambios en el nombre, pronombre y expresión de género, que le permite a la persona presentarse socialmente y en todos los contextos con un género diferente del asignado al nacer (Kuvalanka et al., 2014). Cuando la familia permite realizar una transición social, puede ser un proceso increiblemente afirmativo para las (os, es) niñas (os, es) y adolescentes (Kuvalanka et al., 2014), mostrándoles apoyo en su identidad de género (Sherer, 2016) y generando desafíos para cada una(o) de las (os) miembros de la familia, ya que una transición nunca es un proceso individual (Dierckx et al., 2015).

A pesar de que la transición social es cada vez más común para las (os, es) niñas (os, es) y adolescentes con incongruencia de género (Durwood et al., 2017), se sabe poco acerca de los beneficios que genera en la salud mental de ellas (os, es) (Durwood et al., 2017; Sherer, 2016). Se ha visto que permite un mejor funcionamiento de aquellas(os) niñas (os, es) y adolescentes que se encuentran intensamente disfóricos y además les ayuda a explorar la vida en otro género antes de comenzar cualquier intervención médica (Sherer, 2016), facilitándoles un periodo de introspección y exploración de género (Turban \& Keuroghlian, 2018).

En general la transición requiere apoyo en diversos ámbitos, psicológico, social, médico, quirúrgico y legal (Turban \& Keuroghlian, 2018). Y desde un enfoque afirmativo se debe intervenir no sólo con las (os, es) niñas (os, es) y adolescentes, sino también con las familias, las escuelas y la comunidad, quienes ayudan y favorecen el proceso (Kaltiala-Heino et al., 2018).

\section{Definiciones}

El enfoque afirmativo con niñas (os, es) y adolescentes considera que todas las identidades de género son igualmente válidas, promoviendo la transición social en las (os, es) que lo deseen (Turban et al., 2018). En el proceso se debe intervenir en los ámbitos individual y contextual (Edwards-Leeper et al., 2016), considerándolo un proceso individualizado, comunicado con la (el) niña (o, e) y destacando la posibilidad de una de-transición (Turban \& Keuroghlian, 2018). A continuación, se describen las definiciones en relación al proceso de transición social (Tabla 1). 
Retamal et al.

Tabla 1: Definiciones relacionadas al enfoque transafirmativo y proceso de transición social.

\begin{tabular}{|c|c|}
\hline Concepto & Definición \\
\hline Sexo & $\begin{array}{l}\text { Los determinantes genéticos, anatómicos y hormonales del sexo, clasificados como mujeres, hombres o inde- } \\
\text { terminados. }\end{array}$ \\
\hline Género asignado al nacer & $\begin{array}{l}\text { Hace referencia al género del recién nacido, generalmente declarado por un profesional de la medicina (Turban } \\
\text { et al., 2018). }\end{array}$ \\
\hline Identidad de género & $\begin{array}{l}\text { Se refiere al sentido de la persona de ser mujer, hombre o pertenecer a otro género; por ejemplo, género fluido, } \\
\text { agénero, no binario, otro (Turban et al., 2018). }\end{array}$ \\
\hline Expresión de género & $\begin{array}{l}\text { Hace referencia a la forma en que la persona se presenta al mundo exterior de una forma diferenciada según el } \\
\text { género, incluye el uso de pronombres, vestimenta y otros marcadores externos del género (Turban et al., 2018). }\end{array}$ \\
\hline Incongruencia de género & $\begin{array}{l}\text { Marcada y persistente discordancia entre el género experimentado de la persona y el sexo asignado (Organización } \\
\text { Mundial de la Salud, 2018). }\end{array}$ \\
\hline Transgénero & $\begin{array}{l}\text { Se refiere a una persona en que el sexo experimentado no coincide estrictamente con el género asignado al } \\
\text { nacer (Turban et al., 2018). }\end{array}$ \\
\hline Cisgénero & $\begin{array}{l}\text { Hace referencia a las personas cuyo género experimentado coincide con el género asignado al nacer (Turban } \\
\text { et al., 2018). }\end{array}$ \\
\hline Disforia de género & Malestar psicológico que experimenta la persona en relación con su identidad de género (Turban et al., 2018). \\
\hline Hombre transgénero & $\begin{array}{l}\text { Una persona transgénero que se identifica como masculino, con género asignado al nacer femenino (Turban } \\
\text { et al., 2018). }\end{array}$ \\
\hline Mujer transgénero & $\begin{array}{l}\text { Una persona transgénero que se identifica como femenina, con género asignado al nacer masculino (Turban et } \\
\text { al., 2018). }\end{array}$ \\
\hline Transición social & Presentación en los contextos sociales con su género afirmado (Martinerie et al., 2018). \\
\hline De-transición & Consiste en volver a identificarse con el género asignado al nacer (Turban \& Keuroghlian, 2018). \\
\hline
\end{tabular}

Para el propósito de esta revisión bibliográfica y sugerencias de intervención en transición social con niñas (os, es) y adolescentes se utilizará la clasificación diagnóstica CIE-11 (Organización Mundial de la Salud, 2018) y los términos incongruencia de género y/o transgénero para referirse respecto de esta población infanto juvenil.

El objetivo de este trabajo, es por una parte realizar una revisión teórica de la literatura existente en relación a la transición social en niñas (os, es), adolescentes y además entregar sugerencias a las(os) profesionales respecto del apoyo en la intervención de esta etapa.

\section{Metodología}

El presente trabajo corresponde a una revisión teórica entre los años 2010 - 2021 en revistas científicas de habla inglesa e hispana indexadas en PUBMED, SciELO y en Google Académico. De los artículos encontrados, se revisó además la bibliografía de ellos, sumando a la revisión las publicaciones nacionales que fueran pertinentes (figura 1).

\section{Identificación}

La búsqueda de los artículos fue realizada por todas (os) las (os) autoras (es) utilizando los siguientes términos: transición social en niñas (os, es), adolescente transgénero, gender incongruence in child, social transition in transgender child and youth, social transition in transgender and family, desist and persist in children.

\section{Selección}

Las(os) autoras(es) pre-seleccionaron por abstract, considerando los siguientes criterios: (a) que incorporaran aspectos de transición social en niñas (os, es) y adolescentes, (b) que describieran familias con hijas (os, es) en transición y (c) que describiera intervenciones afirmativas con niñas (os, es) y adolescentes. Se incluyeron además artículos nacionales recientes y dos estudios de organizaciones nacionales LGBTIQ+.

\section{Resultados}

En la selección de artículos, se observó que la incongruencia de género en niñas (os, es) y adolescentes está muy poco desarrollada en habla hispana, con un 16,7\% de los artículos y específicamente el proceso de transición social es inexistente. En habla inglesa se observa un mayor abordaje del proceso de transición social (20\%) y familiar (15\%), específicamente en países como E.E.U.U, Canadá y Holanda. Los estudios nacionales se centran en población adolescente y adulta y no existen estudios en población prepuberal, transición social y abordaje familiar. 


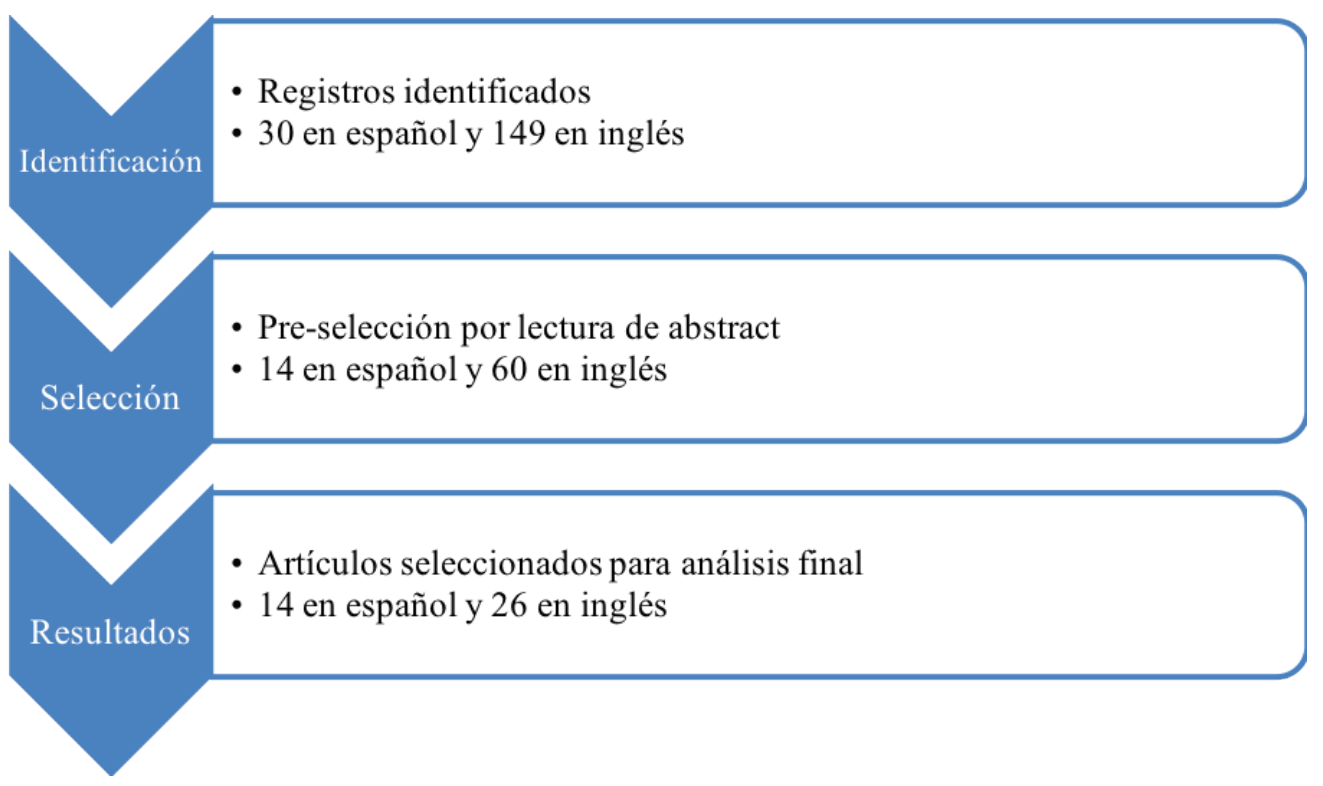

Figura 1: Diagrama de flujo que ilustra el proceso de artículos seleccionados para revisión

\section{Resultados}

\section{Transición social y desarrollo}

Antes de comenzar el proceso de transición social, las (os, es) niñas (os, es) y adolescentes han vivenciado un largo camino en el descubrimiento de su identidad de género. En algunas (os, es) niñas (os, es) este proceso comienza en la temprana infancia en donde reportan una marcada preferencia por actividades, comportamientos y sociabilidad relacionada a la identidad de género experimentada (Espinoza et al., 2019).

A medida que transcurre el crecimiento físico, alrededor de los 10 a 13 años (Steensma et al., 2011), se manifiesta la incomodidad con el desarrollo de los caracteres sexuales primarios y secundarios (Espinoza et al., 2019), activándose interrogantes e incertidumbre acerca de sí misma (o, e), lo que lleva a que se instale un sentimiento de extrañeza y confusión que es vivido con alta tensión emocional (Espinoza et al., 2019). Los cambios corporales llevan a que se produzca un aumento de la disforia o la angustia, trayendo consigo una aversión hacia sus cuerpos, inseguridad y aislamiento social (Steensma et al., 2011). 2011).

El estado de confusión de las niñas (os, es) y adolescentes, instala la necesidad de buscar información relacionada con la orientación sexual hasta llegar a descubrir el concepto transgénero, movilizándolas (os, es) a iniciar los cambios y expresar su identidad (Espinoza et al., 2019). En este periodo algunas (os, es) niñas (os, es) y adolescentes comienzan a realizar transición social parcial, muchas veces sin consentimiento de sus familias.
En la etapa de identificación con la incongruencia de género comienzan a manifestarse síntomas emocionales, autoagresivos y pensamientos suicidas, asociados con el temor a comunicar su identidad, al rechazo y/o discriminación de la familia y pares, junto a la imposibilidad de expresar su género (Espinoza et al., 2019). En esta fase del desarrollo es muy importante el apoyo de profesionales de la salud mental u otras áreas que guien y orienten a la (el) niña (o, e), adolescente y su familia respecto de comenzar un proceso de educación en incongruencia de género y/o de transición social.

\section{Transición social prepuberal}

La transición social en esta etapa del desarrollo es un tema controversial debido a las altas tasas de desistencia que se han publicado (Edwards-Leeper et al., 2016; Kaltiala-Heino et al., 2018; Martinerie et al., 2018; Turban \& Keuroghlian, 2018), pero a pesar de ello la transición social prepuberal puede generar beneficios en salud mental para las (os, es) niñas (os, es) que lo realizan (Turban \& Keuroghlian, 2018).

Algunos estudios han sugerido que cerca del $80 \%$ de las (os, es) niñas (os, es) prepuberes referidos a atención de género posiblemente no se identificarán como personas trans en la adolescencia o adultez (Steensma et al., 2013; Olson, 2016; Turban \& Keuroghlian, 2018). La mayoría de las (os, es) prepuberes se identificarán en el futuro como cisgénero, homosexual (Turban \& Keuroghlian, 2018), lesbiana, bisexual y un pequeño porcentaje como heterosexual (Olson, 2016). Muchos de estos estudios que informaban alta 
desistencia presentaban problemas metodológicos, ya que en la configuración de las muestras incorporaban no solamente a niñas (os, es) con incongruencia de género, sino que también con intereses y expresión de género diferente a su género asignado, de este modo los que persistían era el menor porcentaje o sea el grupo trans (Olson, 2016; Turban \& Keuroghlian, 2018).

Las(os, es) niñas(os, es) que son más propensos a buscar una transición social son aquellas (os, es) con disforia o estrés, es decir las(os, es) Ilamadas(os, es) persistentes (Steensma et al., 2013; Olson, 2016), aquellos que describen su identidad en forma declarativa ("yo soy un niño") más que afectiva ("me gustaría ser un niño") (Steensma et al., 2013; Scherer, 2016; Murchison, 2016) y que insistentemente, persistentemente y consistentemente se identifican con un género distinto del asignado al nacer (Olson, 2016).

En el desarrollo, la identidad de género no es siempre persistente o consistente, sino que también puede presentar una naturaleza dinámica a través del tiempo (Turban \& Keuroghlian, 2018), pudiendo algunas (os, es) niñas (os, es) presentar una de-transición, que consiste en volver a identificarse con el género asignado al nacer (Turban \& Keuroghlian, 2018). Las razones para una de-transición provienen de factores internos de la persona y externos o contextuales. La trayectoria individual del desarrollo del género es un factor interno y el apoyo y/o aceptación por los contextos socio - familiares del tipo externo (Turban \& Keuroghlian, 2018). La de-transición requiere apoyo de profesionales, debiéndose elaborar y utilizar protocolos para el acompañamiento (Turban \& Keuroghlian, 2018).

El enfoque clínico utilizado en los países bajos, respecto del asesoramiento a las (os, es) niñas (os, es) prepúberes, se ha centrado principalmente en tratar las dificultades coexistentes y en realizar un seguimiento del desarrollo del género de la (el) niña (o, e) (Steensma \& Cohen-Kettenis, 2018). En el asesoramiento familiar, se las (os) motiva a apoyar la experiencia de género de la(el) niña (o, e), se les informa que las decisiones son personalizadas para cada niña (o, e) (Steensma \& Cohen-Kettenis, 2018), teniendo la posibilidad de desistir en el futuro (Steensma \& Cohen-Kettenis, 2018; Turban \& Keuroghlian, 2018).

\section{Transición social diferencial en niñas y niños}

En el proceso de transición es importante tener presente las diferencias entre mujeres y hombres con incongruencia de género, ya que en investigaciones realizadas con adultos (Jellestad et al., 2018) se ha informado que los hombres trans tienen un mejor funcionamiento social y salud mental que las mujeres trans, esta diferencia se atribuye a cómo la sociedad trata a las mujeres y hombres trans (Jellestad et al., 2018). Las mujeres trans deben obtener una buena aprobación de su contexto social (Jellestad et al., 2018), están expuestas a alto prejuicio familiar y laboral (Guzmán-González et al.,
2020), en cambio los hombres trans son tratados con más libertad por esos mismos contextos (Jellestad et al., 2018).

En la infancia también se observan algunas diferencias, las cuales son expuestas en un estudio que evaluó la autopercepción en niñas y niños con incongruencia de género, en donde los niños trans eran quienes se percibían mejor en la escuela y en los deportes, se encontraban más satisfechos y más competentes que las niñas trans; situación que podría deberse a que es más aceptado el comportamiento masculino en las niñas, que el femenino en los niños (Alberse, 2019).

Estas diferencias entre niñas y niños, podría tener implicacias en el manejo clínico, debido a que los niños trans tienen una mayor tendencia a la persistencia, alta varianza de género y mayor edad al momento de consultar en un servicio de asistencia en género (Steensma et al., 2013). La situación de que los niños trans consulten más tarde puede estar indicando que ciertos factores serían significativos a diferentes edades para niñas y niños con incongruencia de género y por lo tanto consultar en distintos periodos de tiempo (Steensma et al., 2013), es así que las niñas trans tenderían a una mayor intensidad de la incongruencia de género, lo que las llevaría a realizar transición social más tempranamente (Steensma et al., 2013) y por lo tanto a consultar antes en su desarrollo.

\section{Transición social y salud mental}

La población con incongruencia de género presenta un alto riesgo de enfrentar problemas de salud mental y de experimentar una disminución de la calidad de vida (Organizando trans diversidades, 2017; Jellestad et al.,2018; MOVILH, 2018), debido al estrés que perciben por ser víctimas de prejuicio sexual y social (Barrientos et al., 2019; Guzmán-González et al., 2020). En población adulta con incongruencia de género, se ha reportado una prevalencia de depresión (40\%), diez veces mayor que la población general a nivel mundial (4,4\%) (Guzmán-González et al., 2020), ideación suicida informada en el último año (68\%) que supera el 2,2\% a nivel nacional y lo mismo con los intentos suicidas (34,1\% versus 0,7\%) (Guzmán-González et al., 2020).

La salud mental de las niñas (os, es) también presenta dificultades y sintomatología, particularmente ansiedad, depresión y suicidalidad (Olson et al., 2016; Turban et al., 2018). Estudios muestran que la psicopatología del tipo internalizante como depresión y ansiedad es hasta tres veces mayor en niñas (os, es) con incongruencia de género que no han iniciado un proceso de transición social (Olson et al., 2016; Turban, 2017; Wong \& Drake, 2017). Por el contrario, cuando se inicia se observan resultados positivos, tal como en el estudio de Olson et al., (2016) con un grupo de prepúberes de 3 a 12 años en transición social, en donde se evaluó ansiedad y depresión, mostrando que la sintomatología depresiva en las (os, es) niñas (os, es) transgénero no difería de los grupos controles y la ansiedad era sólo levemente 
mayor. Resultados confirmados en el estudio de Durwood et al., (2017) que evaluó similar sintomatología en reportes de familias y de niñas (os, es) de 9 a 14 años que se encontraban en transición social.

Muchas familias son guiadas por profesionales de la salud mental en la transición social de sus hijas (os, es), observando que con este proceso las (os, es) niñas (os, es) y adolescentes que han presentado una intensa disforia o estrés, frecuentemente se estabilizan en conducta y ánimo (Sherer, 2016), disminuyendo la ansiedad (Kuvalanka et al., 2014), mostrándose más sociables y comprometidas (os, es) (Aramburu, 2018).

Estos hallazgos sugieren que la transición social y el apoyo familiar necesario para iniciarla, favorecen la salud mental de las (os, es) niñas (os, es) y adolescentes con incongruencia de género (Olson et al., 2016; Wong \& Drake, 2017), pueden ser factores protectores de la depresión y ansiedad, junto a estar asociados a una alta satisfacción con la vida (Wong \& Drake, 2017; Hillier \& Torg, 2019). Cuando se es parte de una familia y ambiente que apoya, se observa resiliencia y adecuada salud mental (Tomicic et al., 2016; Aramburu, 2018; Barrientos et al., 2019) y por el contrario el rechazo familiar aumenta a ocho veces más la probabilidad de que un adolescente con incongruencia de género intente suicidarse (Tomicic et al., 2016).

\section{Transición social y familia}

Las familias poseen sus propias normas y expectativas, las cuales han sido creadas a través de la continuidad de las interacciones, pero cuando un miembro de la familia manifiesta conductas que se encuentran fuera de las normas establecidas, las dinámicas relacionales se alteran y las expectativas se desestabilizan (Aramburu, 2018). Desde la teoría de sistemas familiares, en un proceso de transición se deben abordar las necesidades de las madres, padres y cuidadores así como las necesidades de las(os, es) hijas(os, es), debido a que la transición de una(o, e) niña(o, e) y adolescente con incongruencia de género requiere que las familias renegocien su nueva identidad, replanteen adecuadas relaciones familiares (Hillier \&Torg, 2019) y creen nuevos significados y percepciones (Wagner \& Armstrong, 2020), con el objetivo de crear una estabilidad (Aramburu, 2018) que traerá consigo un crecimiento personal para toda la familia (Aramburu, 2018).

Muy poca investigación se ha focalizado en las experiencias familiares (Kuvalanka et al., 2014; Dierckx et al., 2015; Aramburu, 2018) y cómo intervenir en el apoyo a las madres, padres y cuidadores (Hillier \& Torg, 2019). Pese a ello, existen estudios que han descrito las distintas reacciones en las familias de hijas (os, es) con incongruencia de género (Tabla 2). Conocer estos antecedentes les permite a las(os) profesionales tener información valiosa para idear intervenciones adecuadas y efectivas en la labor de apoyo afirmativo.

Tabla 2: Reacciones de las familias al enfrentar la transición de una hija (o, e) transgénero.

\begin{tabular}{|c|c|}
\hline Kuvalanka et al., 2014 & $\begin{array}{l}\text { Vivencia de shock } \\
\text { Alivio por saber lo que sucedía con su hija. } \\
\text { Preocupación por sus hijas con incongruencia de género, especialmente respecto a su seguridad. } \\
\text { Experimentar una transformación desde un desconocimiento de la incongruencia de género hasta educarse en la temática, } \\
\text { permitiendo esto avanzar más rapidamente hacia la aceptación de sus hijas. }\end{array}$ \\
\hline Dierckx et al., 2015 & $\begin{array}{l}\text { Dolor de que su hija (o, e) cambie a alguien diferente de quien ellas (os) habían previsto. } \\
\text { Verguenza derivada de la estigmatización } \\
\text { Vivencia de pérdida } \\
\text { Percepción de responsabilidad a causa de su estatus de madre o padre. } \\
\text { Confusión en los padres respecto de lo que es normal y lo que no lo es. } \\
\text { Sentimiento de culpa a causa de los juicios sociales, lo que las(os) lleva a sentirse calificados como una mala madre o mal } \\
\text { padre cuando aceptan la identidad de su hija (o, e). } \\
\text { Reacciones negativas y rechazo hacia su hija (o, e). } \\
\text { Conflictos de pareja entre ambos padres en relación a las decisiones que cada uno adopta. }\end{array}$ \\
\hline Aramburu, 2018 & $\begin{array}{l}\text { Confusión } \\
\text { Creer que es sólo una etapa. } \\
\text { Preocupación y finalmente convicción de apoyar a su hija (o, e). } \\
\text { Sentimientos de culpa por no haber reconocido el género de su hija(o) a tiempo y evitar el daño o sufrimiento de ella o el. }\end{array}$ \\
\hline Espinoza et al., 2019 & $\begin{array}{l}\text { Consultar a profesionales para intentar corregir la expresión de género de su hija (o, e). } \\
\text { Insistir que es una confusión y un estado transitorio de las (os, es) hijas (os, es). } \\
\text { Buscar ayuda profesional de salud mental y fundaciones para recibir apoyo en este proceso. } \\
\text { Actitud restrictiva activa (retos y golpes) o pasiva (distracción de intereses) ante la expresión de género de su hija (o, e). }\end{array}$ \\
\hline Wagner \& Armstrong, 2020. & $\begin{array}{l}\text { Vivencia de pérdida } \\
\text { Dolor } \\
\text { Impotencia } \\
\text { Miedo acerca de la seguridad de la(el) hija (o, e). } \\
\text { Miedo acerca del futuro de la(el) hija (o, e). }\end{array}$ \\
\hline
\end{tabular}


El proceso de aceptación de la identidad de género de la (el) hija $(\mathrm{o}, \mathrm{e})$ en una familia atraviesa por varias etapas, en donde primeramente la familia descubre y habla de la identidad de género de la(el) hija (o, e). El conocer la identidad de género da paso a un estado de confusión en las(los) miembros de la familia, de bastantes preguntas y cuestionamientos. En esta etapa es muy importante el asesoramiento, la educación y los grupos de ayuda, ya que así se puede establecer el siguiente estado de negociación entre las(os) miembros de la familia para comenzar la adaptación y el equilibrio. El equilibrio se logra cuando la(el) hija (o, e) deja de ocultar su identidad y es integrada (o, e) a la familia (Kuvalanka et al., 2014). Este proceso de aceptación genera un impacto positivo en el ánimo de la(el) hija (o, e) y también en las madres, padres, hermanas(os) y miembros de familia extensa (Kuvalanka et al., 2014), lo que las(os) refuerza como familia en sus decisiones (Aramburu, 2018).

Al interior de una familia, todas (os) las(os) miembros reaccionan y vivencian el proceso de un modo diferente. Las madres han sido descritas como adecuadamente involucradas, así en el estudio de Kuvalanka et al., (2014) respecto de familias de niñas trans, se observó a las madres avanzar desde un shock inicial hasta comprender lo que sucedía con su hijo (a, e), para finalmente apoyar el proceso (Kuvalanka et al., 2014).

Los padres por su parte, a diferencia de las madres, al parecer necesitan más tiempo para aceptar y entender a su hija (o, e) y en ocasiones han sido descritos como con baja participación y conocimiento de la situación (Kuvalanka et al., 2014). Es necesario más estudios al respecto, ya que los padres pueden tener diferentes experiencias en relación a la parentalidad o pueden estar involucrados diferentes significados en el proceso de aceptación y duelo (Kuvalanka et al., 2014).

La edad de las (os) hermanas (os) al momento del proceso de transición puede influir en las reacciones experimentadas, en donde las (os) más pequeñas(os) pueden vivenciar positivamente la identidad de género de su hermana (o, e), pero los de mayor edad pueden percibirlo como una pérdida (Kuvalanka et al., 2014) y por lo tanto iniciar un proceso de duelo.

En este proceso de transición y aceptación, las familias necesariamente deben ser acompañadas (Aramburu, 2018). Dentro de las necesidades de apoyo, se encuentra el comunicarse con personas que hayan experimentado una situación similar y poder compartir experiencias (Kuvalanka et al., 2014; Dierckx et al., 2015). Estos espacios de acompañamiento pueden ser grupales, donde se puede dar la oportunidad de aprender sobre asuntos legales, medicos, escolares y recibir apoyo emocional (Hillier \& Torg, 2019).

\section{Transición social en niñas, niños, niñes y adolescentes en Chile}

Cada vez más en Chile las personas con incongruencia de género y específicamente las niñas (os, es) y adolescentes pueden expresar su identidad de género. A nivel legislativo, en diciembre de 2018 se publicó la ley de identidad de género 21120 (Congreso nacional de Chile, 2018) que reconoce y da protección a la identidad de género. Las (os, es) adolescentes mayores de 14 y menores de 18 años, por medio de su representante legal pueden solicitar en un Tribunal de Familia competente la rectificación del sexo y nombre con el que aparezcan individualizadas (os, es) en su partida de nacimiento y una vez alcanzada la mayoría de edad podrán requerir una nueva rectificación.

En el área sanitaria, el año 2011 se instruye a los establecimientos de la red asistencial con la circular 34 (MINSAL, 2011), respecto a la atención de personas con incongruencia de género en relación con la identificación o nombre social, registro en ficha clínica y hospitalización. En 2012 se da a conocer la circular 21 (MINSAL, 2012) que rige a la fecha y que reitera y profundiza instrucciones sobre la atención de personas con incongruencia de género en la red asistencial respecto de la atención ambulatoria abierta, atención primaria y de especialidades. En la atención sanitaria en el año 2019, el Hospital las Higueras comienza con intervenciones transafirmativas con niñas (os, es) y adolescentes (Retamal et al., 2021). Estas instrucciones $y$ atenciones a nivel salud han permitido que esta población infanto juvenil sea reconocida (os, es) en su identidad, favoreciendo la afirmación de género

A nivel educacional, en el año 2017 se da a conocer la circular 0768 (MINEDUC, 2017), la cual está dirigida a todos los establecimientos educacionales del país públicos y privados, enfocándose en los derechos las niñas (os, es) y adolescentes, particularmente en el uso del nombre social, uniforme adecuado a su identidad de género y uso de baños. Esta circular representa un avance importante en los procesos de transición social y afirmación de género, permitiéndole a la (el) niña (o, e) y adolescente ir ampliando su reconocimiento más allá de su familia.

En Chile la transición social en la mayoría de las (os, es) niñas (os, es) comienza en la adolescencia (32,3\% a 37,8\%) y un menor porcentaje $(2,9 \%$ a $3,7 \%)$ en la etapa prepuberal (Organizando trans diversidades, 2017; MOVILH, 2018). Estos antecedentes nos muestran que en general las (os, es) adolescentes expresan tardíamente su identidad de género, cuando sus caracteres sexuales secundarios ya estan establecidos, lo que puede traer consigo dificultades de salud mental como ansiedad, depresión y suicidalidad. 


\section{Recomendaciones a profesionales en apoyo a la transición social en niñas (os, es), adolescentes y sus familias}

Las recomendaciones que se exponen a continuación fueron seleccionadas desde la literatura revisada y algunas inferidas desde los estudios con niñas (os, es), adolescentes y familias.

1. Las(os) profesionales de la salud mental deben apoyar a las familias en las decisiones respecto a la transición social, facilitando la comunicación entre madres, padres, cuidadores e hijas (os, es) (Martinerie et al., 2018). Es importante promover una respuesta de cuidado, aceptación y apoyo por parte de la familia (Martinerie et al., 2018).

2. Se sugiere que un buen punto de partida para realizar una transición social sería un diálogo con la(el) niña (o, e) y adolescente con el objetivo de comprender sus deseos y ayudarla (o, e) a diferenciar entre expresión e identidad de género. Una vez que una(un) niña (o, e) y adolescente ha comenzado un proceso de transición la comunicación debe mantenerse, observando el desarrollo de la expresión e identidad de género (Olson, 2019).

3. Las(os) profesionales pueden incentivar una transición social parcial, es decir cambio de ropa y cabello determinado a su identidad de género, o una transición total que incorpore pronombres y nombre social, en conjunto con informar a las(os) otras(os) miembros de la comunidad acerca de la situación de las (os, es) niñas (os, es) y adolescentes (WPATH, 2012).

4. Las familias pueden motivar el uso de ropa neutral, particularmente al inicio de la transición social (Aramburu, 2018).

5. Las(os) profesionales pueden motivar una transición social gradual (en casa primero), lo que puede ayudar a reducir la sintomatología ansiosa y depresiva. Estos avances en salud mental incentivan el comenzar a realizar la transición a nivel escolar y en la comunidad en general (Kuvalanka et al., 2014; Edwards-Leeper et al., 2016).

6. Las(os) profesionales pueden plantear la transición social como el experimentar vivir en otro género y en un ambiente seguro, facilitando una experiencia positiva en las (os, es) niñas (os, es) y adolescentes. Se pueden recomendar propuestas intermedias como realizar cambios sólo durante las vacaciones (WPATH, 2012) o transiciones públicas en otras ciudades, en donde la $(o, e)$ niña (o, e), adolescente y la familia no sean conocidas (os, es). Las familias pueden comunicar a las (os, es) niñas (os, es) y adolescentes acerca de las probables reacciones negativas de las demás personas y cómo poder enfrentarlas (Kuvalanka et al., 2014).
7. Es importante que las familias informen a sus hijas (os, es) de forma explícita que una vez iniciada la transición social pueden cambiar su decisión y por lo tanto volver al género asignado al nacer u otro (WPATH, 2012; Olson, 2019).

8. Para disminuir las preocupaciones acerca del futuro de sus hijas(os), las familias pueden incentivar en ellas (os, es) estrategias de afrontamiento, fomento de autonomía y confianza en sí mismas (os, es) (Aramburu, 2018; Alberse, 2019).

9. Es importante que las(os) profesionales ayuden a las familias a incorporar un lenguaje que permita a las (os, es) niñas (os, es) explorar su género en el futuro (por ejemplo, "cuando tengas 16 años comenzarás estrógeno") y que refuerce todas las trayectorias de identidad potenciales (Edwards-Leeper et al., 2016).

10. En la transición con prepúberes también es importante ayudar a las familias a poder convivir con la incertidumbre respecto del futuro en la trayectoria de la identidad de género de la (el) niña (o, e) (Steensma \& Cohen-Kettenis, 2018).

11. Es importante tener en cuenta que las decisiones son personalizadas para cada niña (o, e) y adolescente, siendo lo más adecuado plantear todas las potenciales posibilidades (Steensma \& Cohen-Kettenis, 2018).

12. En la intervención es importante incorporar experiencias grupales, ya que las familias reportan resultados positivos (Hillier \& Torg, 2019).

13. A nivel escolar, en ocasiones se requiere que la (el) niña $(o, e)$ y adolescente se traslade a un nuevo establecimiento educacional que les permita expresar su identidad de género (Espinoza et al., 2019). Es fundamental incluir intervenciones escolares y así facilitar la adaptación y evitar la deserción (Espinoza et al., 2019).

\section{Discusión y conclusiones}

Dentro del enfoque transafirmativo con niñas (os, es) y adolescentes, la transición social se presenta como una intervención no médica, reversible y con un alto beneficio en la salud mental de las (os, es) niñas (os, es) con incongruencia de género. Es importante y necesario apoyar a estas familias respecto de educación, el momento y grado de ir realizándolo.

Lamentablemente existe muy poca literatura en habla inglesa y es inexistente en habla hispana y se evidencia la escasa información existente en nuestro país respecto de incongruencia de género en infancia, adolescencia y particularmente del proceso de transición social. La gran mayoría de la evidencia proviene de equipos que investigan e intervienen en E.E.U.U., Canadá y Holanda, por lo que 
esperamos que las recomendaciones realizadas sean de utilidad, ya que muchas(os) profesionales de la salud mental no tienen la experticia en incongruencia de género, pero igualmente se ven enfrentadas(os) a realizar orientación a estas familias. Es importante educarse, ya que un adecuado apoyo puede influir en disminuir trastornos ansiosos, depresivos y suicidalidad en estas (os, es) niñas (os, es) y adolescentes.

Se debe incorporar el modelo ecológico de intervención, en donde la transición social de la(el) niña (o, e) y adolescente va a generar cambios en la familia y la comunidad. El apoyo de las(los) profesionales debe ir encaminado no sólo a la transición de la(el) niña (o, e) y adolescente, sino también a la propia transición familiar, escolar y comunitaria. De gran relevancia para la familia son los grupos de apoyo, en donde comparten experiencias y se identifican con otras personas que viven situaciones similares. En el equipo transpediátrico del Hospital las Higueras se han realizado actividades grupales, con muy buenos resultados y avances en el proceso de aceptación familiar (Retamal et al., 2021). A nivel escolar, es muy importante educar a las(os) profesores, ya que son ellas(os) quienes en ocasiones pesquisan y derivan a las familias a consultar con un equipo especializado. Desde nuestra experiencia se ha realizado educación en colegios, observando un gran interés y respeto frente a la temática, generando en muchos de ellos cambios en relación a la educación en género que se imparte (Retamal et al., 2021).

La transición social prepuberal tiene bastantes detractores, pero es acogida positivamente cuando se plantea a la familia y a las (os, es) niñas (os, es) desde la posibilidad de explorar otro género o desde los beneficios en la salud mental. La voz de la(el) niña (o, e) es muy relevante en este proceso y es un cambio que como sociedad debemos realizar e ir incorporando cada vez más la opinión de ellas (os, es) en las decisiones. Es necesario destacar que no existen planes de transición iguales para todas (os, es) las (os, es) niñas (os, es), se trata de una intervención individualizada, es decir lo que es adecuado para una (o, e), tal vez para otra $(0, e)$ no sea beneficioso aún. Desde la experiencia del equipo transpediátrico del Hospital las Higueras, el porcentaje de población prepuberal atendida es menor (Retamal et al., 2021) y la experiencia con las familias de estas (os, es) niñas (os, es) nos informa que efectivamente adoptan las sugerencias encaminadas primeramente a realizar transiciones parciales, observando cambios positivos en la salud mental de su hija (o, e) que las (os, es) lleva luego a una transición total.

Importante también a tener en cuenta es el desarrollo diferencial entre niñas y niños trans, ya que en la práctica clínica se observa el trato diferente desde la sociedad, siendo más sancionadora con las conductas femeninas en una niña y adolescente trans, observando que muchas veces la transición social se retrasa debido a estos factores. Así como se ha observado trato diferencial en niñas y niños trans, sería importante evaluar en futuras líneas de revisión e investigación el trato y desarrollo de infancia y adolescencia trans sumado a otras condiciones tales como el espectro autista, el cual se presenta con frecuencia en esta población.

En Chile, ha ido cada vez en aumento la derivación y consulta de niñas (os, es) y adolescentes en equipos especializados en incongruencia de género del área infanto adolescente, sin embargo estamos en deuda aún, ya que las (os, es) niñas (os, es) llegan muy tarde en su desarrollo a realizar la transición social, muchas veces retrasadas (os, es) por las(os) mismas (os) profesionales, las (os) que se refieren a la incongruencia de género como un estado de confusión o una etapa, lo que conlleva problemas de salud mental que se pueden evitar si se realiza a tiempo y con apoyo adecuado de profesionales bien capacitados.

\section{Contribuciones y reconocimientos}

No existe conflicto de intereses de participación como activistas de organizaciones LGBTIQ+, tampoco el trabajo recibió financiamiento.

\section{Referencias}

Alberse A, De Vries A, Elzinga W. \& Steensma T. (2019). Sel-perception of transgender clinic referred gender diverse children and adolescents. Clinical Child Psychology and Psychiatry 24, 388-401.

Aramburu Ch. (2018). Supporting families of transgender children/ youth: parents speak on their experiences, identity and views. International Journal of Transgenderism 19, 132-143.

Barrientos J, Espinoza-Tapia R, Meza P, Saiz J, Cárdenas M, Guzmán-González M, Bahamondez J \& Lovera L. (2019). Efectos del prejuicio sexual en la salud mental de las personas transgénero chilenas desde el modelo de estrés de las minorías: una aproximación cualitativa. Terapia Psicológica 37, 181-197.

Congreso nacional de Chile. (2018). Ley de identidad de género 21120. Accedido en http://www.congreso.cl/ el 11 de febrero de 2021.

Dierckx M, Motmans J, Mortelmans D. \&T'sjoen G. (2015). Families in transition: a literature review. International Review of Psychiatry 28, 1-8.

Durwood L, McLaughlin K. \& Olson K. (2017). Mental health and self - worth in socially - transitioned transgender youth. Journal of the American Academy of Child \&Adolescent Psychiatry 56, 116-123.

Edwards-Leeper L, Leibowitz S. \& Sangganjanavanich V. (2016). Affirmative practice with transgender and gender nonconforming youth: expanding the model. Psychology of Sexual Orientation and Gender Diversity 3, 165-172. 
Ehrensaft D, Giammattei S, Storck K, Tishelman A. \& Keo-Meier C. (2018). Prepubertal social gender transitions: what we know; what we can learn - a view from a gender affirmative lens. International Journal of Transgenderism 59, 251-268.

Espinoza M, Fernández O, Riquelme N. \& Irarrázabal M. (2019). La identidad transgénero en la adolescencia chilena: experiencia subjetiva del proceso. Psykhe 28, 1-12.

Guzmán-González M, Barrientos J, Saiz J, Gómez F, Cárdenas M, Espinoza-Tapia R, Bahamondez J, Lovera L. \& Giami A. (2020). Salud mental en población transgénero y género no conforme en Chile. Revista Médica de Chile 148, 1113-1120.

Hillier A. \& Torg E. (2019). Parent participation in a support group for families with transgender and gender-nonconforming children: "being in the company of others who do not question the reality of our experience". Health Transgender 4, 168- 175.

Jellestad L, Jäggi T, Corbisiero S, Schaefer D, Jenewein J, Schneeberger A, Kuhn A. \& García D. (2018). Quality of life in transitioned trans persons a retrospective cross-sectional cohort study. Journal of Biomedicine Research and Biotechnology 3, 1-10.

Kaltiala-Heino R, Bergman H, Tyolajavi M. \& Frisen L. (2018). Gender dysphoria in adolescence: current perspective. Adolescent Health, Medicine and Therapeutics 9, 31-41.

Kuvalanka K, Weiner J. \& Mahan D. (2014). Child, family, and community transformations: findings from interviews with mothers of transgender girls. Journal of GLBT family studies 10, 354-379.

Martinerie L, Condat A, Bargiacchi A, Bremont-Weill C, De Vries A. \& Hannema S. (2018). Approach to the management of children and adolescents with gender dysphoria. European Journal of Endocrinology 179, 219-237.

MINEDUC (2017). Circular 0768. MAT: Derechos de niños, niñas y estudiantes trans en el ámbito de la educación. Accedido en https://www.supereduc.cl/wp-content/uploads/2017/04/ ORD-N\%C2\%BA0768-DERECHOS-DE-NI\%C3\%91AS-NI\%C3\%91OS-Y-ESTUDIANTES-TRANS-EN-EL-\%C3\%81 MBITO-DE-LA-EDUCACI\%C3\%93N-A-SOSTENEDORES.pdf el 20 de febrero de 2021.

MINSAL (2011). Circular 34. Accedido en http://www.movilh.cl/ documentacion/trans/Circular-Salud-Trans.pdf el 21 de febrero de 2021.
MINSAL (2012) Circular 21. Accedido en http://diprece.minsal.cl/ wrdprss_minsal/wp-content/uploads/2015/01/CIRCULAR-21-Reitera-Atenci\%C3\%B3n-de-personas-trans.pdf el 20 de febrero de 2021. MOVILH (2018). Encuesta identidad. Accedido en http://www.movilh. cl/wp-content/uploads/2018/07/Encuesta_Identidad_-Movilh-2018. pdf el 21 de febrero de 2021.

Murchison G. (2016). Suporting and caring for transgender children. American Academy of Pediatrics. Sept. Accedido en https://assets2. hrc.org/files/documents/SupportingCaringforTransChildren.pdf el 19 de noviembre de 2020.

Olson K, Durwood L, DeMeules M. \& McLaughlin K. (2016). Mental health of transgender children who are supported in their identities. Pediatrics 137, e20153223.

Olson K. (2016). Prepubescent transgender children: what we do and do not know. Journal of the America Academy of Child \& Adolescent Psychiatry 55, 155-6.e3.

Olson K, Blotner Ch, Alonso D, Lewis K, Edwards D. \& Durwood L. (2019). Discussions of early childhood social transitions. Clinical Practice in Pediatric Psichology 7, 229-240.

Organización Mundial de la Salud (2018). Clasificación internacional de enfermedades CIE-11. Accedido en https://icd.who.int/es el 10 de noviembre de 2020.

Organizando trans diversidades (2017). Informe ejecutivo encuesta T. Santiago, Chile: autor. extraído de encuesta-t.cl. Accedido https:// otdchile.org/wp-content/uploads/2020/05/Informe_ejecutivo_Encuesta-T.pdf el 4 de febrero de 2021.

Rae J, Gülgöz S, Durwood L, DeMeules M, Lowe R, Lindquist G. \& Olson K. (2019). Predicting early-childhood gender transitions. Psychological Science 30, 1-13.

Retamal D, Hermosilla W, Arancibia M, Medel M \& Retamal C. (2021). Incongruencia de género en niñas, niños y adolescentes: intervenciones transafirmativas en Hospital las Higueras Talcahuano. ARS MEDICA Revista de Ciencias Médicas 46, 44-54.

Sherer I. (2016). Social transition: supporting our youngest transgender children. Pediatrics 137, e20154358.

Steensma T, Biemond R, De Boer F. \& Cohen-Kettenis P. (2011). Desisting and persisting gender dysphoria after childhood: a qualitative follow-up study. Clinical Child Psychology and Psychiatry16, 499-516. 
Steensma T, McGuire J, Kreukets B, Beekman A. \& Cohen-Kettenis P. (2013). Factors associated with desistence and persistence of childhood gender dysphoria: a quantitative follow up study. Journal of the American Academy of Child and Adolescent Psychiatry 52, 582-590.

Steensma T. \& Cohen-Kettenis P. (2018). A critical commentary on "a critical commentary on follow-up studies and "desistence" theories about transgender and gender non-conforming children". International Journal of Transgender 19, 225-230.

Tomicic A, Gálvez C, Quiroz C, Martínez C, Fontbona J, Rodríguez J, Aguayo F, Rosenbaum C, Leyton F \& Lagazzi I. (2016). Suicidio en poblaciónes lesbiana, gay, bisexual y trans: revision sistemática de una década de investigación (2004-2014). Revista Médica de Chile 144, 723-733.

Turban J. (2017). Transgender youth: the building evidence base for early social transition. Journal of the America Academy of Child \&Adolescent Psychiatry 56, 101-102.

Turban J. \& Keuroghlian A. (2018). Dynamic gender presentations: understanding transition and "de - transition" among transgender youth. Journal of the American Academy of Child \& Adolescent Psychiatry 57, 451-453.
Turban J, De Vries A, Zucker K. \& Shandianloo S. (2018). Transgénero y jóvenes no conformes con su género. Manual de salud mental infantily adolescente de la IACAPAP. Ginebra: Asociación internacional de psiquiatría del niño y el adolescente y profesiones afines. cap. 3 ed. Irarrázabal M, Martin A. trad. Ortega, Espinoza M. Accedido en https://iacapap.org/content/uploads/H.3-GENDER-IDENTITY-Spanish-2018.pdf el 19 de diciembre de 2019.

Wagner L. \& Armstrong E. (2020). Families in transition: the lived experience of parenting a transgender child. Journal of family Nursing 26, 337-345.

Wong W. \& Drake S. (2017). A qualitative study of transgender children with early social transition: parent perspectives and clinical implications. International Journal of Social Sciences 3, 1970-1985.

World professional association for transgender health (WPATH) (2012). Normas de atención para la salud de personas trans y con variabilidad de género (versión en español). Accedido en https:// www.wpath.org/media/cms/Documents/SOC\%20v7/SOC\%20 V7_Spanish.pdf el 10 de diciembre de 2019.

Zapata A, Diaz K, Barra L, Maureira L, Linares J. \& Zapata F. (2019). Atención de salud de personas transgéneros para medicos no especialistas en Chile. Revista Médica de Chile 147, 65-72.

Zapata A, Muena C, Quiroz S, Alvarado J, Leppes F, Villalón J \& Pestén D. (2021). Percepción de la atención de salud de personas transgénero en profesionales médicos y médicas del norte de Chile. Revista Chilena de Obstetricia y Ginecología 86, 61-67. 